\title{
Heteroclinic Connections Between Periodic Orbits in Planar Restricted Circular Three Body Problem. Part II
}

\section{Daniel Wilczak $^{1}$, Piotr Zgliczyński ${ }^{2}$}

1 WSB - NLU, Faculty of Computer Science, Department of Computational Mathematics, Zielona 27, 33-300 Nowy Sạcz, Poland. E-mail: dwilczak@wsb-nlu.edu.pl

2 Jagiellonian University, Institute of Computer Science, Nawojki 11, 30-072 Krakow, Poland. E-mail: umzglicz@cyf-kr.edu.pl

Received: 14 September 2005 / Accepted: 14 September 2005

Erratum published online: 5 November 2005 - @ Springer-Verlag 2005

Commun. Math. Phys. 259, 561-576 (2005)

Unfortunately, Fig. 3 on p. 568 was printed incorrectly. The correct version is given below:

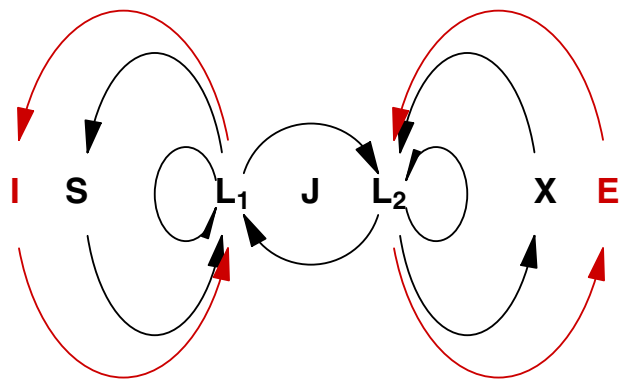

Fig. 3. The graph of symbolic dynamics on six symbols 